\title{
Predictors of Mastoiditis after Intensity-Modulated Ra- diotherapy in Nasopharyngeal Carcinoma: A Dose-Volume Analysis
}

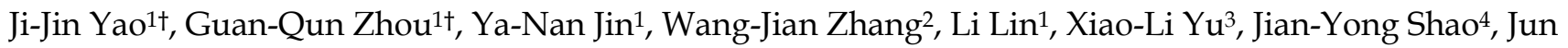
$\mathrm{Ma}^{1}$, Ying Sun ${ }^{1 凶}$

1. Department of Radiation Oncology, Sun Yat-sen University Cancer Center, State Key Laboratory of Oncology in South China, Collaborative Innovation Center for Cancer Medicine, Guangzhou 510060, Guangdong Province, People's Republic of China

2. Department of Medical Statistics and Epidemiology \& Health Information Research Center \& Guangdong Key Laboratory of Medicine, School of Public Health, Sun Yat-sen University, Guangzhou 510080, Guangdong Province, China

3. Department of Radiation Oncology, Sun Yat-sen Memorial Hospital, Sun Yat-sen University, Guangzhou 510120, Guangdong Province, China

4. Department of Pathology, Sun Yat-sen University Cancer Center, State Key Laboratory of Oncology in South China, Collaborative Innovation Center for Cancer Medicine, Guangzhou 510060, Guangdong Province, People's Republic of China

† These authors contributed equally to this manuscript.

$\triangle$ Corresponding author: Ying Sun, Department of Radiation Oncology, Sun Yat-sen University Cancer Center, 651 Dongfeng Road East, Guangzhou, Guangdong 510060, People's Republic of China. Telephone: +86-20-87343816, Fax: +86-20-87343295, Email: sunying@sysucc.org.cn

( ) Ivyspring International Publisher. Reproduction is permitted for personal, noncommercial use, provided that the article is in whole, unmodified, and properly cited. See http://ivyspring.com/terms for terms and conditions.

Received: 2015.07.08; Accepted: 2015.10.29; Published: 2016.01.08

\begin{abstract}
Background: To identify predictors for development of mastoiditis after intensity-modulated radiation therapy (IMRT) in nasopharyngeal carcinoma (NPC).

Methods: Data for 146 NPC patients treated with IMRT was retrospectively reviewed under institutional ethics committee approval. Clinical factors associated with mastoiditis were analyzed. Dose-volume histogram analysis was performed for the Eustachian tube, tympanic cavity, mastoid air cells, cochlea, internal auditory canal and vestibular apparatus to relate doses to radiographic changes in the mastoid. Mastoiditis was assessed using magnetic resonance imaging and was classified as Grade 0 (none), 1 (mild), 2 (moderate) or 3 (severe); Grade 3 mastoiditis was the study end-point.

Results: Eighty-eight ears (36\%) had radiation-induced mastoiditis: 38/244 (15.6\%) mastoid complexes had Grade 1-2 mastoiditis and 50/244 (20.5\%) mastoid complexes had Grade 3 mastoiditis. Multivariate analysis revealed a mastoid mean dose $>35.93$ Gy (odds ratio $[O R]=4.22$, $P=.003)$, Eustachian tube mean dose $>53.43 \mathrm{~Gy}(O R=2.16, P=.034)$ and advanced T category (T3 and T4; $O R=10.33, P=.032$ ) were negative prognostic factors for Grade 3 mastoiditis.

Conclusions: Radiation-induced mastoiditis remains a common late toxicity in NPC after radiotherapy. The mean dose to the mastoid air cells and Eustachian tube should be limited to reduce the risk of radiation-induced mastoiditis.
\end{abstract}

Key words: dose-volume histogram; intensity-modulated radiotherapy; mastoiditis; nasopharyngeal carcinoma; organs at risk.

\section{Introduction}

Nasopharyngeal carcinoma (NPC) is a distinct entity of head and neck cancer, with a specific pathology, epidemiology and treatment modalities. Given the anatomic location and radio-sensitivity of the primary tumor, radiotherapy is usually the primary treatment modality used to achieve local and regional control in NPC ${ }^{1}$. Radiation-induced mastoiditis, characterized by mastoid opacification, ver- 
tigo, tinnitus, hearing loss and other symptoms, is the most common radiotherapy-associated otologic complication associated with $\mathrm{NPC}^{2}$. The incidence of mastoiditis following conventional two-dimensional radiotherapy (2D-CRT) has been reported in several studies and ranges from $15 \%$ to $50 \%{ }^{3-6}$.

Intensity-modulated radiotherapy (IMRT) was a major break-through in the treatment of NPC, and is capable of producing highly conformal dose distributions with steep dose gradients and complex isodose surfaces. The design of appropriate dose constraints for the organs at risk (OARs) during optimization of IMRT treatment plans can significantly reduce subsequent complications. However, knowledge of the dose constraints for particular structures that influence the occurrence of mastoiditis in the IMRT era is lacking to date. There is a critical need for more accurate information regarding dose limits to prevent mastoiditis in patients with NPC receiving IMRT.

Therefore, in this study, a cohort of patients with NPC who did not have mastoiditis before radiotherapy was retrospectively reviewed to evaluate dose-volume histogram (DVH) predictors for mastoiditis. We aimed to identify the incidence of mastoiditis after IMRT and determine how the radiation doses to particular structures influence the risk of mastoiditis.

\section{Materials and Methods}

\section{Study population}

This study included 146 patients with newly-diagnosed, non-distant metastatic, histologically-proven NPC who underwent the entire prescribed course of IMRT at the Sun Yat-sen University Cancer Centre (Guangzhou, People's Republic of China) between January 2009 and April 2010. This retrospective study was conducted in compliance with the institutional policy to protect the patients' private information and was approved by the institutional review board of Sun Yat-Sen University Cancer Center.

All patients completed a pre-treatment evaluation that included a physical examination, nasopharyngeal and neck magnetic resonance imaging (MRI) scan, chest radiography, abdominal sonography and whole body bone scan. Additionally, 23 patients (15.9\%) underwent positron emission tomography computed tomography (PET-CT). Medical records and imaging studies were retrospectively analyzed, and all patients were restaged according to the seventh edition of the Union for International Cancer Control/American Joint Committee on Cancer (UICC/AJCC) staging system ${ }^{7}$. Patients with evidence of distant metastasis at diagnosis were excluded from the study.

\section{MRI techniques}

MRI was performed using a 1.5-Tesla system (Signa CV/i; General Electric Healthcare, Chalfont St. Giles, United Kingdom) using a head-and-neck combined coil to examine the area spanning the suprasellar cistern to the inferior margin of the sternal end of the clavicle. T1-weighted fast spin-echo images in the axial, coronal and sagittal planes (repetition time, 500-600 ms; echo time, 10-20 ms), and T2-weighted fast spin-echo MRI in the axial plane (repetition time, 4000-6000 ms; echo time, 95-110 ms) were obtained before injection of contrast material. Following intravenous injection of gadopentetate dimeglumine $(0.1$ $\mathrm{mmol} / \mathrm{kg}$ body weight Gd-DTPA, Magnevist; Bayer-Schering, Berlin, Germany), spin-echo T1-weighted axial and sagittal sequences and spin-echo T1-weighted fat-suppressed coronal sequences were performed sequentially. Diffusion-weighted MRI using line scan diffusion images was performed with a pelvic phased-array coil with $b$-values of 5 and $800 \mathrm{~s} / \mathrm{mm}^{2}$. Section thickness was 5 $\mathrm{mm}$ with a $1 \mathrm{~mm}$ interslice gap for the axial plane, and $6 \mathrm{~mm}$ with a $1 \mathrm{~mm}$ interslice gap for the coronal and sagittal planes.

\section{Image evaluation}

Image evaluation was performed independently by two radiologists and one clinician, each with more than 10 years' experience in head and neck cancer. If there was disagreement, consensus was reached by discussion. The diagnosis of mastoiditis was made in accordance with the criteria proposed by Platzek et $a l .{ }^{8}$. The volume measurement of mastoid opacification was based on our previously reported proposals 9 , and mastoiditis was divided into four grades according to the criteria of Walker et al. ${ }^{4}$.

\section{Radiotherapy and chemotherapy}

Each patient received IMRT, which covered the primary tumor to the upper neck area above the caudal edge of the cricoid cartilage. Target volumes were delineated using our institutional treatment proto$\mathrm{Col}^{10}$, in accordance with the International Commission on Radiation Units and Measurements reports 50 and 62. The prescribed doses were: total dose of 68-70 Gy in 30-33 fractions at 2.13-2.27 Gy/fraction to the planning target volume (PTV) of the GTV-P (i.e. primary gross tumor volume), 60 Gy to the PTV of CTV-1 (i.e. high-risk regions), 54 Gy to the PTV of CTV-2 (i.e. low-risk regions and neck nodal regions), and 60-68 Gy to the nodal GTV in 30-33 fractions. Treatment was delivered once daily, over five fractions per week.

During the study period, the institutional guidelines recommended no chemotherapy for patients with stage I disease and concurrent chemoradiother- 
apy \pm neoadjuvant/adjuvant chemotherapy for patients with stage II-IVB disease, as defined by the sixth edition of the UICC/AJCC staging system for NPC. Twelve patients (12/146; 8.2\%) with stage III/IV disease that were scheduled to receive chemotherapy received only radiotherapy alone; the reasons for deviation from guidelines were old age, organ dysfunction, or allergic reactions that indicated intolerance to chemotherapy. Overall, $21 / 146$ patients $(14.4 \%)$ were treated with radiotherapy alone, and $125(85.6 \%)$ received chemotherapy. Neoadjuvant or adjuvant chemotherapy consisted of cisplatin with 5-fluorouracil and/or docetaxel every three weeks for two or three cycles. Concurrent chemotherapy consisted of cisplatin administered weekly or on days 1 , 22 and 43 of radiotherapy.

\section{Contouring of the auditory apparatus and data collection}

The volumes of the components of the auditory apparatus: the Eustachian tube (ET), tympanic cavity (TC), mastoid air cells, vestibular apparatus (VS), cochlea and internal auditory canal (IAC) were contoured on every slice by a radiation oncologist. Based on anatomic definitions, a reasonable contouring method for the auditory apparatus (Figure 1) was employed ${ }^{11}$. Dose-volume histograms (DVH) were generated to determine the mean dose to each component of the auditory apparatus. Additionally, one treatment-related variable (concurrent chemotherapy) and patient-related variables (age, patient sex, $\mathrm{T}$ classification, $\mathrm{N}$ classification and pathologic features) were included as clinical variables. A total of 13 variables were reviewed.

\section{Follow-up and statistical analysis}

Follow-up was calculated from the first day of therapy to the day of either death or last examination. Patients were followed-up at least every three months in the first three years and every six months thereafter. Routine follow-up care included a complete head and neck examination, hematology and biochemistry profiles, chest radiography and abdominal sonography. Follow-up neck and/or nasopharynx MRI were performed every 6-12 months, especially for cases with suspected tumor recurrence or radiotherapy-induced complications.

All analyses were performed using R3.1.2. Associations between potential risk factors and the occurrence of Grade 3 mastoiditis were evaluated. Receiver operating characteristic (ROC) curves were generated to select the optimal cut-points for the mean dose to each component of the auditory apparatus in patients with and without severe mastoiditis. The area under the ROC curve (AUC) reflects the ability of the test to distinguish between individual ears with and without severe mastoiditis. The predictive value of each DVH parameter was evaluated based on the AUC. Predictors with an AUC significantly higher than 0.5 were then transformed into binary variables according to the optimal cut-off points selected and incorporated into univariate logistic regression models. Finally, a multivariate logistic regression model was used to identify predictors that have a significant impact on Grade 3 mastoiditis. The Nemenyi-test with Chi-squared approximation was applied for multiple comparisons. A $P$-value $<0.05$ was considered statistically significant for all tests.

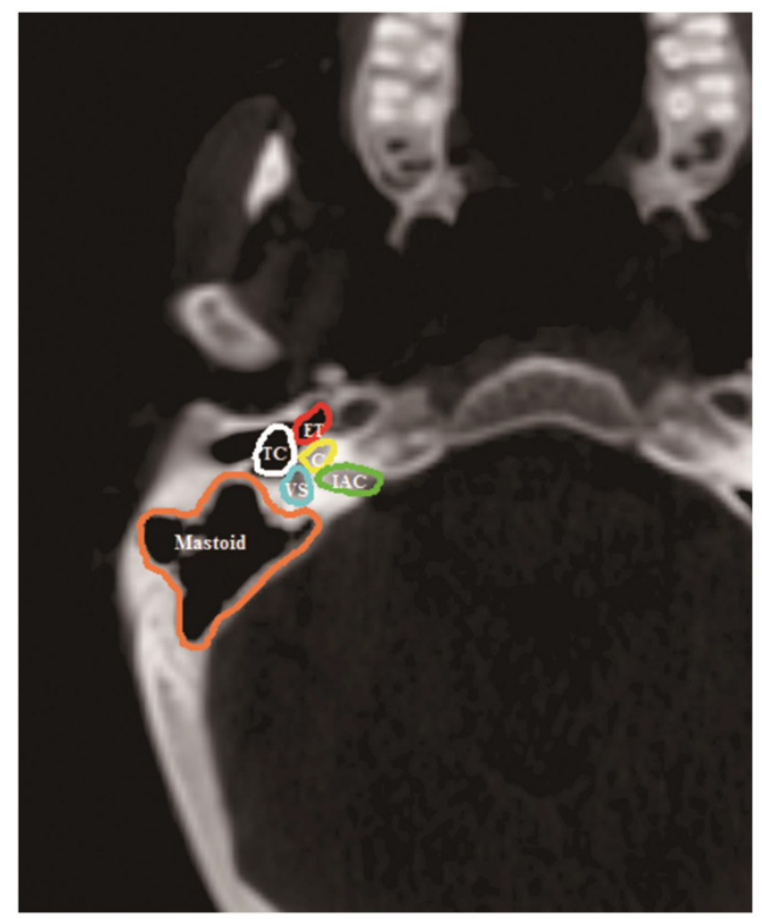

Figure 1. CT anatomy of the Eustachian tube (ET), tympanic cavity (TC), mastoid air cells (Mastoid), vestibular apparatus (VS), cochlea (C), and internal auditory canal (IAC).

\section{Results}

The left and right ears were evaluated separately, and patients with mastoid effusion at pre-treatment MRI were excluded. Overall, a total of 146 patients (244 ears) were enrolled in this retrospective study. The median follow-up time for this cohort of patients was 43 months (range, 31-50 months), and the final follow-up was performed on January 15, 2015. The 4-year overall survival rate was $85.6 \%$ and the local recurrence-free rate was $93.2 \%$.

\section{Patient characteristics}

The characteristics of the 146 patients (244 ears) are summarized in Table 1 . The male/female ratio was 2.7:1 (106 males, 40 females); median age was 44.6 
years (range, 14-63 years). Histologically, all patients were diagnosed with type 1 or 2 disease according to World Health Organization (WHO) guidelines: $10 / 146(0.7 \%)$ of cases had WHO type 1 disease and $136 / 146(99.3 \%)$ of cases had WHO type 2 disease. Forty-seven patients (32.2\%) had T1/T2 disease and 99/146 (67.8\%) had T3/T4 disease. Sixty-six patients (45.3\%) had N0/N1 disease and 80/146 (54.8\%) had N2/N3 disease. Twenty-three patients $(15.8 \%)$ were diagnosed with stage I or II NPC and 123 patients $(84.2 \%)$ were diagnosed with stage III or IV NPC.

Table 1. Characteristics of the 146 patients ( 244 ears) with NPC

\begin{tabular}{|c|c|c|}
\hline Characteristic & No. & $\%$ \\
\hline \multicolumn{3}{|l|}{ Age, years } \\
\hline$<50$ & 109 & 74.7 \\
\hline$\geq 50$ & 37 & 25.3 \\
\hline \multicolumn{3}{|l|}{ Gender } \\
\hline Male & 106 & 72.6 \\
\hline Female & 40 & 27.4 \\
\hline \multicolumn{3}{|l|}{ Pathological type } \\
\hline WHO Type 1 & 10 & 0.7 \\
\hline WHO Type 2 & 136 & 99.3 \\
\hline \multicolumn{3}{|l|}{ T category* } \\
\hline $\mathrm{T} 1$ & 15 & 10.3 \\
\hline $\mathrm{T} 2$ & 32 & 21.9 \\
\hline $\mathrm{T} 3$ & 55 & 37.7 \\
\hline $\mathrm{T} 4$ & 44 & 30.1 \\
\hline \multicolumn{3}{|l|}{ N category* } \\
\hline No & 23 & 15.8 \\
\hline N1 & 43 & 29.5 \\
\hline N2 & 62 & 42.5 \\
\hline N3 & 18 & 12.2 \\
\hline \multicolumn{3}{|l|}{ Stage group* } \\
\hline I & 6 & 4.2 \\
\hline II & 17 & 11.6 \\
\hline III & 72 & 49.3 \\
\hline IVA-B & 51 & 34.9 \\
\hline \multicolumn{3}{|l|}{ Chemotherapy } \\
\hline CRT & 125 & 85.6 \\
\hline RT alone & 21 & 14.4 \\
\hline \multicolumn{3}{|l|}{ Grades of mastoiditis } \\
\hline G0M & 156 & 63.9 \\
\hline G1M & 28 & 11.5 \\
\hline G2M & 10 & 4.1 \\
\hline G3M & 50 & 20.5 \\
\hline
\end{tabular}

Abbreviations: NPC=Nasopharyngeal carcinoma; IMRT=Intensity modulated radiotherapy; $\mathrm{WHO}=$ World Health Organization; $\mathrm{T}=\mathrm{Tumor} ; \mathrm{N}=$ Node;

$\mathrm{CRT}=$ Chemo-radiationtherapy; $\mathrm{RT}=$ Radiation therapy; $\mathrm{G} 0 \mathrm{M}=\mathrm{Grade} 0$ mastoiditis G1M=Grade 1 mastoiditis; G2M=Grade 2 mastoiditis; G3M=Grade 3 mastoiditis. *According to the 7th American Joint Committee on Cancer/International Union Against Cancer staging system. the G3M group (Grade 3 mastoiditis after radiotherapy) containing 50/244 (20.5\%) mastoid complexes. The incidence of subperiosteal abscesses was $10 \%$ $(5 / 50)$ in the G3M group at the last follow-up MRI according to the criteria reported by Polat et al ${ }^{12}$.

The incidence of G1-2M and G3M was $17 / 85$ (20\%) and 14/85 (16.5\%) in patients with T1-2 disease and $21 / 159(13.2 \%)$ and $36 / 159(22.6 \%)$ in the patients with T3-4 disease, respectively. The incidence of G1-2M and G3M was 23/105 (21.9\%) and 18/105 $(17.1 \%)$ in patients with N0-1 disease and 15/139 (10.8\%) and 32/139 (23\%) in patients with T2-3 disease. The incidence of G1-2M and G3M was 12/59 $(20.3 \%)$ and $13 / 59(22 \%)$ in patients aged $>50$ years and $23 / 185(12.4 \%)$ and $37 / 185(20 \%)$ in patients aged $\leq 50$ years. The incidence of G1-2M and G3M was $8 / 35(22.9 \%)$ and $42 / 209(20.1 \%)$ in patients that received chemoradiotherapy and $9 / 35(25.7 \%)$ and $29 / 209(13.9 \%)$ in patients that received radiotherapy alone.

\section{Univariate/multivariate analysis}

A total of 13 parameters (see Materials and Methods) were analyzed in the current study, including both clinical variables and dosimetric parameters. ROC curve analysis was performed to select the optimal cut-off points for the dosimetric parameters for ears with or without G3M. The results of the ROC curve analysis are shown in Table 2. The optimal cut-off points for the mean dose to the mastoid, cochlea, ET, IAC, TC and VS were 35.93 Gy, 48.86 Gy, 53.43 Gy, 46.49 Gy, 41.13 Gy and 42.10 Gy, respectively.

Table 2. ROC curve analysis for the auditory apparatus in the individual ears of patients with NPC who developed Grade 3 mastoiditis

\begin{tabular}{|c|c|c|c|c|c|c|}
\hline \multirow[t]{2}{*}{ Variable } & \multirow{2}{*}{$\begin{array}{l}\text { Area under } \\
\text { ROC curve }\end{array}$} & \multicolumn{2}{|l|}{$95 \% \mathrm{CI}$} & \multirow[t]{2}{*}{ Cutoff (Gy) } & \multirow[t]{2}{*}{ Sensitivity } & \multirow[t]{2}{*}{ Specificity } \\
\hline & & Lower & Upper & & & \\
\hline Mastoid & 0.67 & 0.59 & 0.75 & 35.93 & 0.38 & 0.91 \\
\hline Cochlea & 0.66 & 0.57 & 0.74 & 48.86 & 0.54 & 0.76 \\
\hline ET & 0.65 & 0.56 & 0.74 & 53.43 & 0.58 & 0.68 \\
\hline IAC & 0.62 & 0.53 & 0.72 & 46.49 & 0.58 & 0.74 \\
\hline $\mathrm{TC}$ & 0.69 & 0.60 & 0.76 & 41.13 & 0.70 & 0.60 \\
\hline VS & 0.63 & 0.54 & 0.72 & 42.10 & 0.40 & 0.86 \\
\hline
\end{tabular}

Abbreviations: $\mathrm{ROC}=$ Receiver operating characteristic; $\mathrm{CI}=$ Confidence interval; $\mathrm{NPC}=$ Nasopharyngeal carcinoma; ET=Eustachian tube; IAC=Internal auditory canal; TC=Tympanic cavity; VS=Vestibular apparatus.

According to the optimal cut-points determined from the ROC curves, each component of the auditory apparatus was transformed into a binary variable. The results of univariate analyses are shown in Table 3. A mastoid mean dose > $35.93 \mathrm{~Gy}$, IAC mean dose > $46.49 \mathrm{~Gy}$, cochlea mean dose $>48.86$ Gy, ET mean dose > 53.43 Gy, TC mean dose > 41.13 Gy, VS mean dose $>42.10$ Gy and advanced T category (T3 and T4)
1-2 mastoiditis after radiotherapy) oro containing 38/244 (15.6\%) mastoid complexes, and 
were significantly associated with G3M. In multivariate analysis, a mastoid mean dose $>35.93$ Gy (OR [odds ratio] $=4.22,95 \%$ confidence interval $[\mathrm{CI}]=$ $[1.63,11.28], P=.003)$, ET mean dose $>53.43$ Gy $(O R=$ $2.16,95 \% C I$ for $O R=[1.06,5.69], P=.034)$ and advanced $\mathrm{T}$ category $(O R=10.33,95 \% C I$ for $O R=[1.78$, 198.51], $P=.032$ ) emerged as independent predictors of severe mastoiditis (Table 3 ).

\section{Subgroup analysis of DVH parameters inde- pendently associated with G3M}

The analysis above showed that severe mastoiditis was associated with a mastoid mean dose $>35.93$ Gy and ET mean dose > 53.43 Gy. The performance of these factors for the prediction of G3M was determined using ROC curve analysis. The area under the ROC curve (AUC) was 0.67 for a mastoid mean dose > 35.93 Gy $(95 \%$ CI for AUC = [0.59, 0.75]; Figure 2A). From Figure 2A, it would be appropriate to consider a mastoid mean dose $>35.93$ Gy as the dose limit for G3M (sensitivity, 0.38; specificity, 0.91). For the group of ears with a mean mastoid dose $>35.93 \mathrm{~Gy}$, the incidence of G1-2M and G3M was $15.1 \%$ and $22 \%$ compared to $23.1 \%$ and $7.7 \%$ in the group with a mean mastoid dose $\leq 35.93 \mathrm{~Gy}$, respectively. The mean $( \pm$ $\mathrm{SD})$ doses to the mastoid for the groups of ears with G0M, G1-2M and G3M were 31.10 \pm 0.43 Gy, $31.92 \pm$ $0.46 \mathrm{~Gy}$ and $33.99 \pm 0.46 \mathrm{~Gy}$, respectively. Multiple comparisons revealed that the mean dose to the mastoid in G3M group was significantly higher than the G0M group $(P<.001)$.

The AUC was 0.62 for an ET mean dose $>53.43$ Gy $(95 \%$ CI for AUC = [0.53, 0.72]; Figure 2B). From Figure 2B, it would be appropriate to consider an ET mean $( \pm \mathrm{SD})$ dose $>53.43 \mathrm{~Gy}$ as the dose limit of the

Table 3. Summary of univariate \& multivariate analysis for Grade 3 mastoiditis

\begin{tabular}{lllllll}
\hline & \multicolumn{3}{l}{ Univariate analysis } & \multicolumn{3}{l}{ Multivariate analysis } \\
\hline Variable & OR & $95 \%$ CI & $P$ & OR & $95 \%$ CI & $P$ \\
\hline Mastoid mean dose > 35.93 Gy & 5.99 & $2.84-12.80$ & $<0.001$ & 4.22 & $1.63-11.28$ & 0.003 \\
ET mean dose > 53.43 Gy & 3.87 & $2.04-7.47$ & $<0.001$ & 2.16 & $1.06-5.69$ & 0.034 \\
T category (T1-2 vs. T3-4) & 8.47 & $1.55-158.04$ & 0.045 & 10.33 & $1.78-198.51$ & 0.032 \\
Cochlea mean dose >48.86 Gy & 3.78 & $1.98-7.27$ & $<0.001$ & & & \\
IAC mean dose >46.49 Gy & 2.94 & $1.56-5.62$ & 0.001 & & & \\
TC mean dose >41.13 Gy & 3.55 & $1.85-7.10$ & $<0.001$ & & & \\
VS mean dose >42.10 Gy & 4.12 & $2.05-8.30$ & $<0.001$ & & & \\
Age (> 50 years vs. $\leq 50$ years) & 1.01 & $0.48-2.02$ & 0.974 & & & \\
Gender (male vs. female) & 1.81 & $0.93-3.48$ & 0.075 & & & \\
Chemotherapy (yes vs. no) & 0.85 & $0.37-2.12$ & 0.708 & & & \\
N category (N0-1 vs. N2-3) & 1.51 & $0.52-5.07$ & 0.469 & & & \\
Pathology (WHO type 1 vs. & 3.81 & $0.74-69.91$ & 0.202 & & & \\
WHO type 2) & & & & & &
\end{tabular}

ET for G3M (sensitivity, 0.58; specificity, 0.74). In the group of ears with an ET mean dose $>53.43 \mathrm{~Gy}$, the incidence of G1-2M and G3M was $11.3 \%$ and $36.3 \%$ compared to $17.7 \%$ and $18.9 \%$ in the group with an ET mean dose $\leq 53.43 \mathrm{~Gy}$. The mean doses to the ET were $50.97 \pm 0.68 \mathrm{~Gy}, 52.50 \pm 0.62 \mathrm{~Gy}$ and $55.62 \pm 0.82 \mathrm{~Gy}$ for the G0M, G1-2M and G3M groups, respectively. Multiple comparisons revealed that the mean dose to the ET in the G3M group was significantly higher than the G0M group $(P=.003)$.
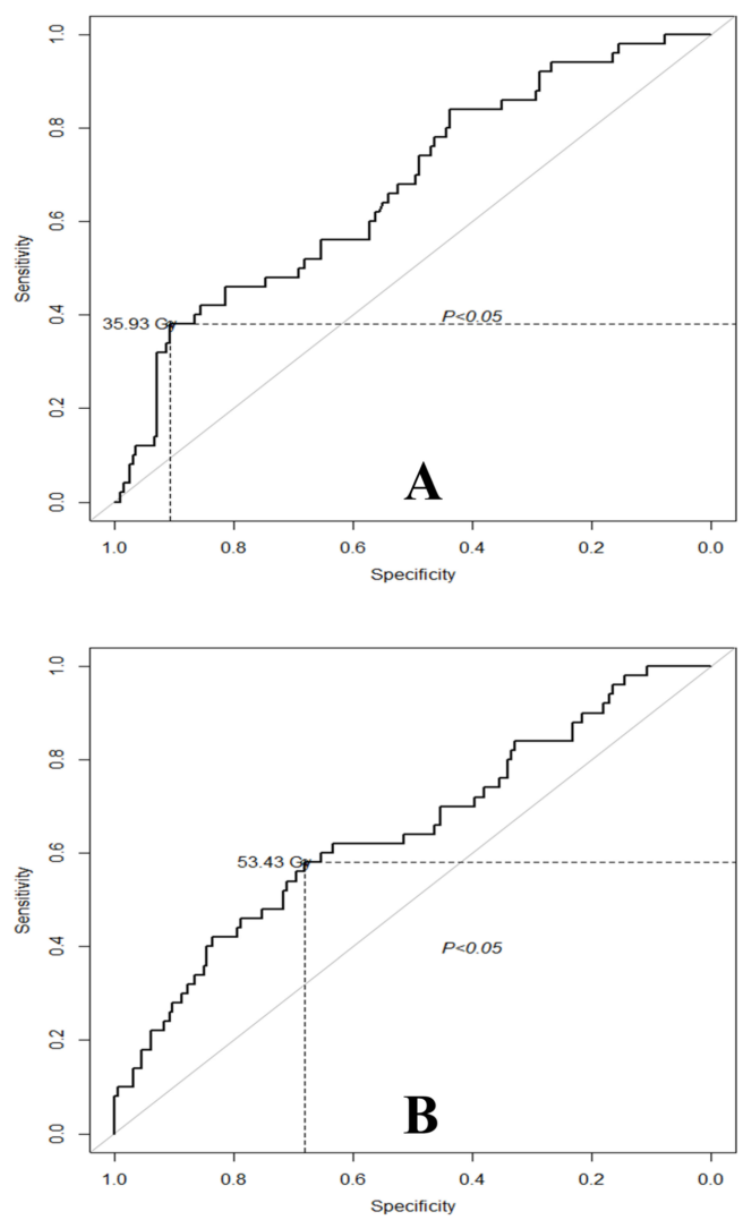

Figure 2. (A) Receiver operating characteristic (ROC) curve analysis for the mean dose to the mastoid with respect to G3M. The cutoff point for the mean dose to the mastoid was determined as 35.93 Gy. (B) ROC curve analysis for the mean dose to the ET with respect to G3M. The cutoff point for the mean dose to the ET was determined as $53.43 \mathrm{~Gy}$.

\section{Discussion}

Radiation-induced mastoiditis is a common complaint in patients with NPC after radiotherapy; however, knowledge of the dose limits for the auditory apparatus with respect to mastoiditis is lacking to date. This retrospective study analyzed the dose-response relationships for mastoiditis, with the purpose of improving the 
understanding of mastoiditis and thus reducing the occurrence of radiation-induced mastoiditis in patients with NPC undergoing IMRT.

\section{Incidence of mastoiditis}

Although NPC can be treated effectively with radiotherapy, many patients complain of permanent mastoiditis after radiotherapy. Nishimura et al. ${ }^{3}$ reported the incidence of radiation-induced mastoiditis was $18 \%, 13 \%$ and $8 \%$ within $6,6-12$ and 12 months after 2D-CRT, respectively. Walker et al. ${ }^{4}$ showed mastoiditis persisted in 23/40 (42.5\%) patients treated using three-dimensional radiotherapy (3D-CRT) (median follow-up, 23 months). In the present study, the incidence of G3M was 23\% (50/244) with the overall incidence of mastoiditis reaching $36.1 \%(88 / 244)$, which is close to the rates reported by Walker et al., and much higher than those reported by Nishimura et al.

Two factors may explain these discrepancies, the first of which is the different diagnostic modalities utilized in each study. The major diagnostic criterion for mastoiditis in the report by Nishimura et al. was the patients' clinical symptoms. However, most cases of mastoiditis are asymptomatic or have very vague symptoms, which may lead to an omission bias and therefore an underestimation of the true incidence of mastoiditis. Considering these shortcomings of earlier works, we used T2W and T1W imaging with contrast enhancement combined with diffusion weighted imaging (DWI) to obtain a higher sensitivity and diagnostic accuracy for mastoiditis. Secondly, although IMRT provides superior escalation of dose coverage to the target, the CTV-2 inevitably includes the medial part of the ET, a critical portion of the ear associated with tubal dysfunction ${ }^{13}$. Therefore, the dose to the cartilaginous portion of the ET may be the same or even higher when using IMRT than 2D-CRT; therefore, IMRT may not decrease the incidence of radiation-induced mastoiditis.

\section{Dose-response relationship for G3M}

Dose-response analyses have been scant to date. Nishimura et al. ${ }^{3}$ retrospectively reviewed 114 patients who underwent 2D-CRT for various diseases of the head and neck using fields that included the temporal bone, and suggested the dose of radiation to the region anterior or posterior to the clival line should be confined to 40-50 Gy to reduce the risk of radiation-induced mastoiditis. More recently, Walker et al. ${ }^{4}$ studied 61 patients treated with 3D-CRT for skull base tumors and suggested the mean dose to the mastoid and posterior nasopharynx should be limited to $<30$ Gy. In this study, a mastoid mean dose > 35.93 Gy and ET mean dose $>53.43$ Gy were significantly associated with G3M in patients with NPC treated with IMRT. The difference between our observations and previous studies may partially be explained by the use of different radiation techniques.

Although it remains unclear how different mechanisms interact to cause mastoiditis in patients with NPC after radiotherapy, this study shows the mean doses to the mastoid and ET are associated with G3M. This finding indicates that radiation-induced destruction of the ET and mastoid plays an important role in the occurrence of radiation-induced mastoiditis. It is likely that damage to the ET and surrounding structures (tensor veli palatini muscle, cartilage, nerves) leads to development of negative pressure in the mastoid, resulting in transudation of serous fluid. This fluid may impede sound conduction through the mastoid and become inoculated with bacteria from the nasopharynx. In addition, direct radiation damage to the mastoid air cell mucosa, blood vessels and lymphatics could induce a non-infectious inflammatory response ${ }^{14}$.

\section{Clinical features as risk factors for G3M}

Clinical variables such as age, gender, pathology, $\mathrm{T}$ category, $\mathrm{N}$ category and treatment with cisplatin were evaluated in this study. Although reports on the association of these factors with mastoiditis have been lacking to date, several previous studies reported that $\mathrm{T}$ category and chemotherapy were associated with otitis media with effusion (OME) in NPC ${ }^{15-18}$. Hsin et al. ${ }^{18}$ showed radiation-induced OME was more likely in patients with advanced T category (T3 and T4). Our data showed that more than $60 \%$ of patients with advanced T category (T3/T4) NPC developed G3M. Both univariate $(P=.045)$ and multivariate $(P=.032)$ analysis indicated advanced T category increased the risk of G3M. This observation may be due to the fact that ET injuries caused directly by the primary tumor may increase the risk of radiation-induced mastoiditis in patients with NPC, as demonstrated by a pathologic study of human temporal bone showing tumor invasion of the paratubal area in patients with head and neck tumors ${ }^{19}$.

Although both cisplatin and radiotherapy may cause ototoxicity ${ }^{20}$, the combined effects of these two factors are unclear. Kwong et al. ${ }^{21}$ prospectively examined patients with NPC treated using radiotherapy and cisplatin, and reported that low-dose cisplatin did not appear to enhance radiation-induced OME. Hitchcock et al..$^{22}$ reported cisplatin ototoxicity may be dose-dependent ${ }^{23}$. This series included patients treated with cisplatin-based chemotherapy. Although the incidence of G3M increased after radiotherapy and chemotherapy compared to radiotherapy alone, chemotherapy had no significant effect on the occur- 
rence of G3M. However, this analysis may be affected by the limited number of patients, as only 21/146 $(14.4 \%)$ patients received radiotherapy alone and the remainder received chemotherapy.

\section{Conclusion}

To our knowledge, this is the first single-institution study to analyze the associations between dose-volume effects and radiation-induced mastoiditis in patients with NPC treated using IMRT. Our data demonstrate patients with a mastoid mean dose $>35.93$ Gy and ET mean dose > 53.43 Gy are at increased risk for mastoiditis. This study provides valuable insight into the risk factors for mastoiditis, and will help to optimize NPC treatment planning to avoid radiation-induced mastoiditis.

\section{Acknowledgments}

This work was supported by grants from the National Natural Science Foundation of China (No. 81372409), the Sun Yat-Sen University Clinical Research 5010 Program (No. 2012011), the Natural Science Foundation of Guangdong Province (No. S2013010012220) and the Science and Technology project of Guangzhou, City, China (No. 132000507). The funders had no role in study design, data collection and analysis, decision to publish, or preparation of the manuscript.

\section{Competing Interests}

The authors have declared that no competing interest exists.

\section{References}

1. Vokes EE, Liebowitz DN, Weichselbaum RR. Nasopharyngeal carcinoma. Lancet.1997; 350: 1087-1091.

2. Kuo CL, Wang $\mathrm{MC}, \mathrm{Chu} \mathrm{CH}$, et al. New therapeutic strategy for treating otitis media with effusion in postirradiated nasopharyngeal carcinoma patients. J Chin Med Assoc. 2012; 75(7): 329-934.

3. Nishimura R, Baba Y, Murakami R et al. MR evaluation of radiation otomastoiditis. Int J Radiat Oncol Biol Phys. 1997; 39 (1):155-160.

4. Walker GV, Ahmed S, Allen P, et al. Radiation-induced middle ear and mastoid opacification in skull base tumors treated with radiotherapy. Int J Radiat Oncol Biol Phys. 2011; 81(5):819-823.

5. Nagorsky, M. J. Radiation injury of the temporal bone. Clin. Plastic Surg. 1993;30:531-534

6. Swart, J. D. Temporal bone inflammatory disease. In: Som, P. M.; Curtin, H. H., eds. Head and neck imaging, 2nd ed. St. Louis, MO: Mosby-Year Book; 1996; 1391-1424.

7. Frederick L et al. Pharynx (Including Base of Tongue, Soft Palate, and Uvula). In: Edge SB, Fritz AG, Byrd DR, Greene FL, Compton CC, Trotti A, editors. AJCC cancer staging manual. New York: Springer. 2010; 41-56.

8. Platzek I, Kitzler HH, Gudziol V, Laniado M, Hahn G. Magnetic resonance imaging in acute mastoiditis. Acta Radiol Short Rep. 2014; 3(2): 2047981614523415

9. Yao JJ, Zhou GQ, Yu XL, et al. Incidence of and Risk Factors for Mastoiditis after Intensity Modulated Radiotherapy in Nasopharyngeal Carcinoma. PLoS One. 2015; 10(6):e0131284.

10. Zhao C, Han F, Lu LX, et al. Intensity modulated radiotherapy for local-regional advanced nasopharyngeal carcinoma. Ai Zheng. 2004 Nov; 23(11):1532-7.

11. Sun $Y, Y u$ XL, Luo W, et al. Recommendation for a contouring method and atlas of organs at risk in nasopharyngeal carcinoma patients receiving intensity-modulated radiotherapy. Radiother Oncol. 2014; 110(3):390-397.

12. Polat S, Aksoy E, Serin GM, et al. Incidental diagnosis of mastoiditis on MRI. Eur Arch Otorhinolaryngol.2011; 268:1135-1138.
13. Kujawski OB, Poe DS. Laser eustachian tuboplasty. Otol Neurotol 2004; 25:1-8.

14. Xu YD, Ou YK, Zheng YQ, et al. The treatment for postirradiation otitis media with effusion: A study of three methods. Laryngoscope 2008; 118:2040-2043.

15. Low WK, Toh ST, Wee J, et al. Sensorineural hearing loss after radiotherapy and chemoradiotherapy: a single, blinded, randomized study. J Clin Oncol. 2006; 24(12):1904-1909.

16. Lee N, Harris J, Garden AS, et al. Intensity-modulated radiation therapy with or without chemotherapy for nasopharyngeal carcinoma: radiation therapy oncology group phase II trial 0225. J Clin Oncol. 2009; 27:3684-3690.

17. Walker DA, Pillow J, Waters KD, et al. Enhanced cis-platinumototoxicity in children with brain tumours who have received simultaneous or prior cranial irradiation. Med Pediatr Oncol. 1989; 17(1):48-52.

18. Hsin $\mathrm{CH}$, Chen $\mathrm{TH}$, Liang $\mathrm{KL}$, et al. Postirradiation otitis media with effusion in nasopharyngeal carcinoma patients treated by intensity-modulated radiotherapy. Laryngoscope. 2013; 123(9): 2148-2153.

19. Yamaguchi N, Sando I, Hashida Y, et al. Histopathologic study of otitis media in individuals with head and neck tumors. Ann Otol Rhinol Laryngol. 1990; 99:827-832.

20. Induction chemotherapy plus radiation compared with surgery plus radiation in patients with advanced laryngeal cancer. The Department of Veterans Affairs Laryngeal Cancer Study Group. N Engl J Med. 1991; 324:1685-1690.

21. Kwong DL, Wei WI, Sham JS, et al. Sensorineural hearing loss in patients treated for nasopharyngeal carcinoma: A prospective study of the effect of radiation and cisplatin treatment. Int J Radiat Oncol Biol Phys. 1996; 36(2):281-289.

22. Hitchcock YJ, Tward JD, Szabo A, et al. Relative contributions of radiation and cisplatin-based chemotherapy to sensorineural hearing loss in head-and-neck cancer patients. Int J Radiat Oncol Biol Phys. 2009; 73(3):779-788.

23. Skinner R, Pearson AD, Amineddine HA, et al. Ototoxicity of cisplatinum in children and adolescents. Br J Cancer. 1990; 61(6):927-931. 